\begin{tabular}{|c|c|c|c|c|c|}
\hline $\begin{array}{l}\text { HIV-1 } \\
\text { isolates }\end{array}$ & & V3 sequences & $\begin{array}{l}\% \text { homology to } \\
\text { cons. B seq. }\end{array}$ & $\begin{array}{l}\text { Positive } \\
\text { charges }\end{array}$ & $\begin{array}{l}\text { Cell } \\
\text { tropism }\end{array}$ \\
\hline B Cons. & CTRPNNNTRK & KS. . IHI . .GPGRAFYTTGEIIGDIRQAHC & 100 & 3 & \\
\hline $\mathrm{BaL}$ & 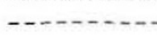 & 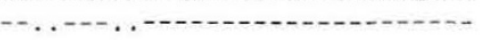 & 100 & 3 & M \\
\hline Ada & $-2-1-n$ & 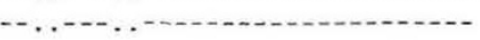 & 100 & 3 & M \\
\hline JRFL & -........... & -- . .--_, & 100 & 3 & M \\
\hline JRCSF & $----s-n-1$ & 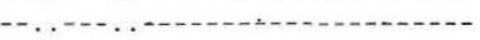 & 97 & 3 & M \\
\hline SF162 & - n.......... & $--\ldots-\mathrm{T}-\ldots-1----\mathrm{A}-\mathrm{D}-\mathrm{D}-\ldots \ldots$ & 91 & 3 & M \\
\hline HXB2 & -non & $-R .,-R-Q R------V-I-K .--N M-\cdots$ & 74 & 9 & $\mathrm{~T}$ \\
\hline LAI & 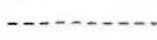 & - IR-QR. . ...---V-I-K---NM-- - & 77 & 8 & $\mathrm{~T}$ \\
\hline NL43 3 & $-\cdots-1-n$ & $--\ldots-R-Q R-\cdots--V-I-K---N M-\cdots$ & 80 & 8 & T \\
\hline MN & $-\ldots--Y-K-$ & -R...-....... & 83 & 8 & $\mathrm{~T}$ \\
\hline $\mathrm{SF} 2$ & $-\ldots-n-1$ & 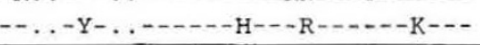 & 89 & 6 & $\mathrm{~T}$ \\
\hline
\end{tabular}

A period (.) represents a deletion; "Location where most $M$-tropic viruses have acidic amino acid residues whereas $T$. tropic strains often have basic residues'. Sequences were aligned according to Myers, G. et al.'.

more complementary negatively charged residues than those of CCR5. Comparing the sequences of these two proteins, it is apparent that the net negative charges in the extracellular domains of CXCR4 are indeed much higher than those of CCR5 (Table 2). This may explain why T-tropic viruses preferentially bind to CXCR4. It seems that in the early stages of HIV-1 infection, most of the primary isolates have M-tropic V3 sequences which preferably bind to CCR5 on $\mathrm{M} / \mathrm{M}$. In contrast, in the later stages, due to the increasing pressure of antibody response against the principal neutralizing domains (PND) in V3 loops, the sequences of V3 loops in gp120 of these viruses have to mutate into T-tropic structures positively charged residues. The outcome of this viral mutation is twofold: First, T-tropic viruses can preferably bind to CXCR4 on T lymphocytes, induce syncytium and become more virulent than M-tropic isolates. Second, T-tropic strains may escape attack by antibodies directed against the M-tropic V3 sequences.

\section{SHIBO JIANG}

Lindsley F. Kimball Research Institute

The New York Blood Center, 310 E. 67th Street,

New York, New York 10021
Table 2 Comparison of net charges in extracelluar domains of CXCR4 and CCR5 $\begin{array}{ll}\text { Extracellular } & \text { Net charges in the domains } \\ \text { domains } & \text { CXCR4 CCR5 }\end{array}$

$\begin{array}{lcc}\text { First } & -6(1-39) & -1(1-30) \\ \text { Second } & +1(100-110) & 0(90-102) \\ \text { Third } & -3(176-200) & +2(167-198) \\ \text { Fourth } & -2(262-285) & 0(261-277) \\ \text { Total } & -10 & +1\end{array}$

The numbers in parentheses are the first and last residues of the named domains (from Swiss-Prot: P30991 and P51681).

1. Cocchi, F. et al. The V3 domain of the HIV-1 gp 120 envelope glycoprotein is critical for chemokine-mediated blockade of infection. Nature Medicine 2, 1244-1247 (1996).

2. De Jong, I. et al. Human immunodeficiency virus type 1 clones chimeric for the envelope $\mathrm{V} 3$ domain differ in syncytium formation and replication capacity. J. Virol. 66, 757-765 (1992).

3. Feng, Y., Broder, C.C., Kennedy, P.E. \& Berger, E.A. HIV1 entry cofactor: Functional CDNA cloning of a seventransmembrane G-protein-coupled receptor. Science 272, 872-877 (1996).

4. Deng, H.K. et al. identification of a major co-seceptor for primary isolates of HN-1. Nature 381, 661-667 (1996).

5. Lapham, C.K. et al. Evidence for cell-association between fusin and the CD4-gp120 complex in human cell lines. Science 274, 602-605 (1996).

6. Wu, L. et al. Induced interaction of HIV-1 gp120 glycoprotein with the chemokine receptor CCR5. Nature 384, 179-183 (1996).

7. Myers, G. et al. Human Retrovirus and AIDS. Los Alamos National Laboratory, Los Alamos, NM (1995).

\title{
Transmission of $L$. infantum by blood donors
}

To the editor - The possible transmission of Leishmania via blood transfusion was raised by the World Health Organization in 1990. We screen blood from donors to the Monaco Blood Bank who live in areas endemic for $L$. infantum, checking for potential asymptomatic Leishmania carriers and searching for parasitemia. To date, sera of 463 donors have been analyzed by western blotting, and 61 of them exhibited 14and/or 18-kDa bands previously shown to correlate with a positive leishmanin skin test'. To date, $L$. infantum promastigotes have been detected by culture in blood samples from nine donors. In some cases detection of parasites was only possible by maintaining cultures for more than three months. The necessity of such long culture periods (observed also in Balb/c infection, unpublished data) might be due to a low concentration of the autocrine growth regulating factor secreted by promastigotes ${ }^{2}$, when the initial parasite load in the culture is low, resulting in slow proliferation. It is interesting that the blood of six (out of nine) culture-positive donors was drawn in April, before the annual emergence of a new generation of Leishmania-transmitting sandflies, indicating that parasitemia may occur long after infection. We also confirmed that the blood of culture-positive donors can transmit Leishmania to Syrian hamsters (injected with high loads of PBMCs purified from the buffy coats).

Although Monaco is an endemic area, it is difficult to prove that leishmaniasis results from a transfusion rather than from the bite of a sandfly.

Our preliminary data show that $L$. infantum, the agent of visceral leishmaniasis and common around the Mediterranean and in Brazil, circulates in the blood of asymptomatic subjects and raise important epidemiological and public health questions.
JOANNA KUBAR, JEAN-FrançoIs Quaranta, Pierre MarTy, Alain Lelièvre \& Yves Le Fichoux Groupe de Recherche en Immunopathologie de la Leishmaniose, Laboratoire de Parasitologie, Faculté de Médecine, 06107 Nice Cedex 2, France

\section{JeAn-Pierre Aufeuvre}

Centre de Transfusion Sanguine, Hôpital Princesse Grace, 98000 Monaco

1. Marty, P. et al. Use of the leishmanin skin test and Western blot analysis for epidemiological studies in visceral leishmaniasis areas: Experience in a highly endemic focus in Alpes-Maritimes (France). Trans. R. Soc. Trop. Med. Hyg. 88, 658-659 (1994).

2. Lemesre, J.L. et ai. Autorégulation de la croissance in vitro des Trypanosomatidae. C.R. Acad. SCi. Paris 307, 283-288 (1988).

We thank Georgette Pagliardini and Sophie Fornasero for their help. 